\title{
Power Amplifier Characteristic-Aware Energy-Efficient Transmission Strategy ${ }^{\star}$
}

Kwanghun Han, Youngkyu Choi, Sunghyun Choi, and Youngwoo Kwon

\author{
School of Electrical Engineering and INMC \\ Seoul National University, Seoul, Korea \\ $\{k h h a n, y k c h o i\} @ m w n l . s n u . a c . k r,\{$ schoi, ykwon\}@snu.ac.kr
}

\begin{abstract}
The energy consumption in transmitting an information bit, i.e., energy-per-bit, has been known to decrease monotonically as the transmission time increases [1. However, when considering the power amplifier (PA) characteristics, we learn that the energy-per-bit starts to increase as the transmission time becomes long over a certain threshold. This is caused by the fact that, in a wireless device, it is not the transmission power, which determines the energy consumed during transmissions, but the input power to the PA whose output power is used as the transmission power. Based on our new trade-off model between the energy-per-bit and transmission time, we revisit known energy-efficient scheduling algorithms. Finally, we evaluate the impact of the new tradeoff model and the performance of algorithms via simulations.
\end{abstract}

\section{Introduction}

For battery-powered hand-held mobile devices, it is a key concern to reduce the energy consumption in order to extend the device's life time. Since the wireless communication module is a major source of the overall energy consumption in such devices 2, 3, many studies in the literature have tried to minimize the energy consumption by turning off the unused parts of the wireless communication module after finishing packet transmission as quickly as possible [4, 5, 6].

In the meantime, the authors in [1,7] proposed another approach, which reduces the transmission energy by controlling the transmit power when sending a given amount of data. They showed based on Shannon's capacity equation that the energy-per-bit monotonically decreases as the transmission time 1 increases. According to this trade-off relation, the authors proposed Lazy scheduling algorithm, which minimizes the transmission energy by sending an information bit as slowly as possible, which is accomplished by using low order modulation, low code rate channel coding and low transmission power. However, their model [1]

\footnotetext{
* This research is in part supported by University IT Research Center (ITRC) project, and by the SNU-Samsung $4 \mathrm{G}$ collaboration project.

${ }^{1}$ Here, the exact meaning of the transmission time is the inverse of spectral efficiency. Assured that it is not confusing, hereafter, we will simply use this term to represent this meaning.
} 
accounts for only the transmission energy while circuitry typically keeps consuming energy in order to maintain the transmission module consistently active even when there is no on-going packet transmission. The power required by circuitry is called electronics power, and this is almost constant irrespective of the transmission power. When considering the effect of electronics power, Yu et al. showed that the energy-per-bit does not monotonically decrease with transmission time any more, especially when the electronics power is comparable with the transmit power [8]. Such a situation can indeed happen when the transmission range is extremely small as in the sensor networks, while the transmit power dominates the electronics power in most wireless communication systems. Shuguang et al. verified through in-depth circuit-level analysis [9] that such a trade-off relation is really present in the context of sensor network. Based on this trade-off model, the authors in [8] proposed a packet scheduling algorithm, which aims at minimizing the energy dissipation in multi-hop wireless sensor networks. Then, in a long-range communication, e.g., conventional cellular network, where the transmit power dominates the electronics power, is it still reasonable to think that the energy-per-bit monotonically decreases as the transmission time increases?

Our major finding, in this paper, is that even if the electronics power is ignorably small compared with the transmit power (i.e., long-range communications), the energy-per-bit does not monotonically decrease as transmission time increases when the power amplifier (PA) characteristics are considered. In a strict sense, we should note that the actual energy consumption is not caused by the transmission energy but by the energy consumed by PA to generate the transmit power. Accordingly, we revisit the energy-efficient scheduling problems, which are considered in [1,10], based on our new trade-off model. We expect that if the algorithms derived in this paper are used for uplink scheduling, especially in a cellular network, the lifetime of battery-powered device could be extended.

The rest of this paper is organized as follows: In Sect. 2, we derive the tradeoff model between the energy-per-bit and transmission time considering the PA characteristics. In Sect. 3, we formulate the energy-efficient scheduling problems, and then present not only two offline algorithms, called Modified Lazy scheduling and Modified Move Right scheduling, but also the online version of each offline algorithm, respectively. In Sect.4, we evaluate the performance of the algorithm via simulation. Finally, in Sect. 5] we conclude the paper with some remarks on the future work.

\section{Revised Trade-Off Model}

The PA efficiency is defined as the ratio of the output power to the power provided by direct current (DC) voltage source. Since the PA output power is equivalent to the transmit power in the context of communication system, we denote the output power by $P_{t x}$ as shown in Fig. 1, 


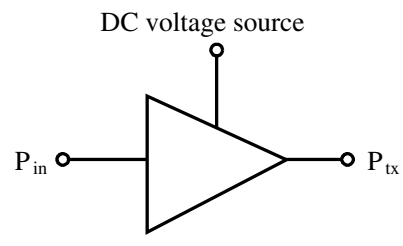

Fig. 1. A diagram for power amplifier

Let us denote the power efficiency by $\eta\left(P_{t x}\right)(<1)$, which is typically nondecreasing with $P_{t x}$ [11,12. Then, $P_{p a}$, which represents the actual power consumed by PA, is represented as

$$
P_{p a}=\frac{P_{t x}}{\eta\left(P_{t x}\right)}, 0 \leq P_{t x} \leq P_{t x, \max },
$$

where $P_{t x, \max }$ is the maximum output power, a design parameter of a PA 2 Due to the dependence of $\eta$ on $P_{t x}$, (1) implies that as we reduce $P_{t x}$ by $\Delta$, the decrement of $P_{p a}$ is not linearly proportional to $\Delta$, but is altered by the operating range of $P_{t x}$.

Under an Additive White Gaussian Noise (AWGN) channel with noise power $N$, the optimal channel coding gives Shannon's capacity as follows:

$$
C=\frac{1}{2} \log _{2}\left(1+\frac{\alpha P_{t x}}{N}\right)
$$

where $\alpha$ represents the power loss due mainly to the path loss. Although the actual transmission rate should be less than $C$, for the convenience of discussion, we just regard $C$ as the achievable rate. When we denote the time necessary to transmit one information bit by $t=\frac{1}{C}$, the energy-per-bit, $E_{r}(t)$, is given by

$$
E_{r}(t)=t P_{p a}=t \frac{P_{t x}}{\eta\left(P_{t x}\right)}, P_{t x}=\frac{N}{\alpha}\left(2^{\frac{2}{t}}-1\right) .
$$

If $\eta\left(P_{t x}\right)$ is simply modeled by a constant, $E_{r}(t)$ is monotonically decreasing and convex in $t$ as shown in [1,7]. Now, using a proposed power efficiency model in [13, we replace $\eta\left(P_{t x}\right)$ with

$$
\eta\left(P_{t x}\right)=\eta_{\max } \sqrt{\frac{P_{t x}}{P_{t x, \max }}},
$$

\footnotetext{
${ }^{2}$ In fact, the additional power provided by PA amounts to $P_{t x}-P_{i n}$, and hence those, who are interested in the PA performance itself, typically use a metric called poweradded efficiency (PAE), which is defined as $\frac{P_{t x}-P_{i n}}{P_{p a}}$. Since the PA gain defined as $\frac{P_{t x}}{P_{i n}}$ typically ranges from 20 to $30 \mathrm{~dB}, P_{t x}-P_{i n} \simeq P_{t x}$, and hence PAE can be thought to be nearly equal to the power efficiency.
} 
where $\eta_{\max }$ is the maximum PA efficiency achieved when $P_{t x}=P_{t x, \max }$. Accordingly, we obtain a new energy-per-bit $E_{r}(t)$ as follows:

$$
E_{r}(t)=\frac{\sqrt{P_{t x} P_{t x, \max }}}{\eta_{\max }} t .
$$

We present two theorems, which show the property of the trade-off relation between $E_{r}(t)$ and $t$. For the purpose of comparison, we denote by $E_{c}(t)$ the energy-per-bit obtained when the power efficiency is considered constant, e.g., $\eta=\eta_{\max }$ as in the literature.

Theorem 1. $E_{r}(t)$ is neither monotonically decreasing nor convex in $t$.

Proof. It can be shown that as $t$ becomes much smaller than $1, E_{r}(t) \sim 2^{\frac{1}{t}} t$ and $E_{c}(t) \sim 2^{\frac{2}{t}} t$. Therefore, $E_{r}(t)$ is also monotonically decreasing and convex in $t$ as $E_{c}(t)$. On the other hand, as $t$ approaches infinity, $E_{c}(t)$ approaches a constant i.e., $\frac{2 N \ln 2}{\alpha \eta_{\max }}$ while $E_{r}(t) \sim \sqrt{t}$. Thus, $E_{r}(t)$ becomes an increasing and concave function in the region of large $t$. Since $E_{r}(t)$ changes from a decreasing convex function to an increasing concave function in the range of $t$ from 0 to infinity, we can prove that there exist both minimum and inflection points of $E_{r}(t)$.

Theorem 2. When we denote $t$ yielding the minimum and inflection points of $E_{r}(t)$ by $t^{*}$ and $t^{o}$, respectively, $t^{*}<t^{o}$.

Proof. From (5), $E_{r}(t)=\beta \sqrt{2^{\frac{2}{t}-1}}$, where $\beta=\frac{\sqrt{N P_{t x, \max }}}{\sqrt{\alpha} \eta_{\max }}$. Differentiating $E_{r}(t)$ by $t$, we obtain $\frac{d E_{r}}{d t}=\beta\left(2^{\frac{2}{t}}-1\right)^{-\frac{1}{2}} g(t)$, where $g(t)=\left(1-\frac{\ln 2}{t}\right) 2^{\frac{2}{t}}-1$. Since $E_{r}(t)$ becomes an increasing function when $t$ is large, $g(t)<0$ when $t<t^{*}$, and $g(t)>0$ when $t>t^{*}$.

Differentiating $\frac{d E_{r}}{d t}$ again, we obtain $\frac{d^{2} E_{r}}{d t^{2}}=\beta\left(2^{\frac{2}{t}}-1\right)^{-\frac{3}{2}} 2^{\frac{2}{t}} \frac{\ln 2}{t^{3}} k(t)$, where $k(t)=(2 \ln 2-1) 2^{\frac{2}{t}}-2 \ln 2$. From $k\left(t^{o}\right)=0, t^{o}=\frac{2}{\log _{2}\left(\frac{2 \ln 2}{2 \ln 2-1}\right)} \simeq 1$ 1.0849. Since $g\left(t^{o}\right) \simeq 0.296>0, t^{*}$ should be less than $t^{o}$. Indeed, a numerical solution for $g(t)=0$ yields $t^{*} \simeq 0.8699$, and hence $t^{*}<t^{o}$ is verified.

Figure 2 plots both $E_{c}(t)$ and $E_{r}(t)$. Referring to the parameters of RF2162PA 13 , we set $P_{t x, \max }$ to $1.41 \mathrm{~W}$ and $\eta_{\max }$ to 0.5 . Assuming that the received signal-to-noise ratio (SNR), $\frac{\alpha P_{t x}}{N}$, ranges from $0.11 \mathrm{~dB}$ to $20 \mathrm{~dB}$, we set $\frac{N}{\alpha}$ appropriately in order that $P_{t x}$ does not exceed $P_{t x, \max }$ at the highest SNR (i.e., $20 \mathrm{~dB}$ ). Obviously, we see that $E_{r}(t)$ becomes quite different from $E_{c}(t)$. This observation tells us that the energy-efficient scheduling algorithm should be revisited based on the new trade-off model. This motivation is differentiated from other approaches [7,8] because the trade-off model, which considers only the effect of electronics power, just accounts for the short-range communication like sensor network, while the electronics power can be ignored in the energy consumption of many other communication systems. 


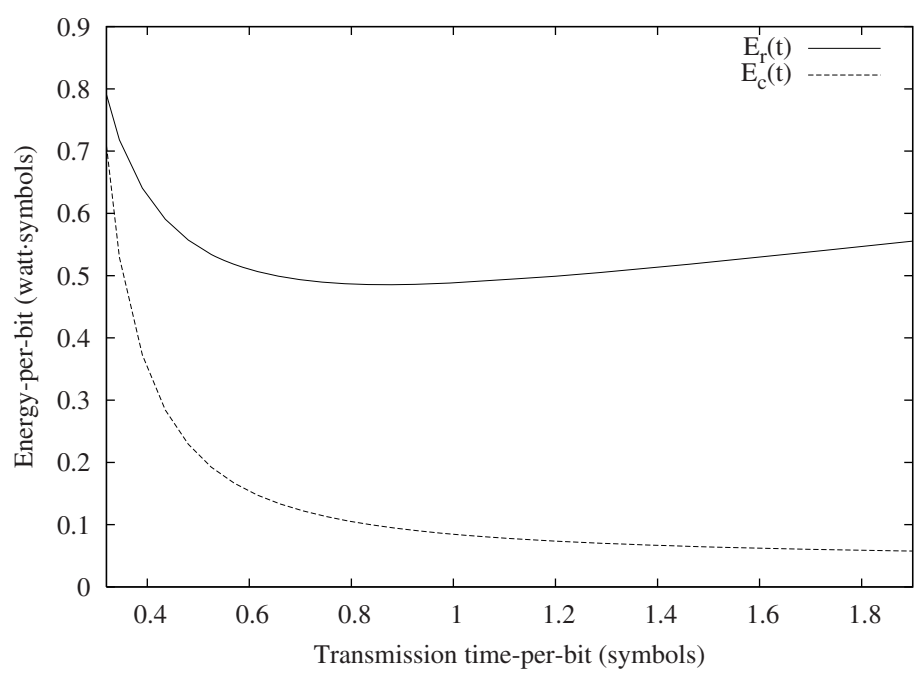

Fig. 2. Energy-per-bit versus transmission time

\section{$3 \quad$ Energy Efficient Uplink Scheduling}

In this section, we consider the energy efficient uplink scheduling problem in cellular systems. Based on our trade-off model, we derive offline and online algorithms.

\subsection{Problem Definition}

In cellular systems, most subscriber stations are battery-powered while the base station is AC-powered. Accordingly, the energy efficient transmission strategy can be beneficial, especially for uplink transmissions. In order to highlight the impacts due to our new trade-off model, we restrict the discussion to the scenario of a point-to-point communication. Let us denote by $w(\tau)$ the amount of energy required to transmit a packet over a time duration $\tau$ by subscriber stations.

We assume that $M$ packets should be transmitted within time $[0, T)$, which can be considered the time allocated to a subscriber station by the scheduler. For the purpose of analytical simplicity, all packets are assumed to be of equal size. We denote the arrival time of the $i$ th packet by $t_{i}$ and the packet interarrival time by $d_{i}=t_{i+1}-t_{i}$. Without loss of generality, the first packet is assumed to arrive at time zero, i.e., $t_{1}=0$. The scheduler determines both the packet transmission duration $\tau=\left(\tau_{1}, \cdots, \tau_{M}\right)$ and the transmission start time $\mathbf{s}=\left(s_{1}, \cdots, s_{M}\right)$. Based on this system description and assumption, we formulate an energy-efficient scheduling problem constrained by the total allowed transmission time for a group of packets, which is equivalent to the problem considered in [1]. 
Problem 1. Find the scheduling, $\tau$ and $\mathbf{s}$, which minimizes the total transmission energy:

$$
\begin{gathered}
\min W(\tau)=\sum_{i=1}^{M} w\left(\tau_{i}\right), \\
\text { s.t. } \\
t_{i} \leq s_{i}, \forall i \in\{1, \ldots, M\}, \\
s_{M}+\tau_{M} \leq T .
\end{gathered}
$$

For the purpose of notation, we denote this problem by ESP-I. As shown in Problem 1, the transmission of the last $M$ th packet should be completed within time $T$. However, since Problem 1 does not take care of the delay, which an individual packet would experience, if $T$ is quite large, the scheduling result may not be desirable for some types of traffic. Note that packets often have transmission delay bound, and hence packets exceeding a certain delay bound would be considered a delivery failure or discarded. This is quite typical for packets out of real-time multimedia applications. Therefore, we consider another problem formulation of energy-efficient scheduling with constraints of per-packet delay bound, and denote the problem by ESP-II.

Given a vector of per-packet delay bound $\mathbf{q}=\left(q_{1}, \cdots, q_{M}\right)$, where $q_{i}$ represents the maximally allowed transmission time for the $i$ th packet, ESP-II can be written as follows:

Problem 2. Find the scheduling, $\tau$ and $\mathbf{s}$, which minimizes the total transmission energy:

$$
\begin{array}{cc} 
& \min W(\tau)=\sum_{i=1}^{M} w\left(\tau_{i}\right), \\
\text { s.t. } & \\
& t_{i} \leq s_{i}, \forall i \in\{1, \cdots, M\}, \\
s_{i}+\tau_{i} \leq q_{i}, \forall i \in\{1, \cdots, M\} .
\end{array}
$$

\subsection{Offline Algorithm}

Modified Lazy Scheduling Algorithm. In order to solve ESP-I, Lazy scheduling algorithm was originally developed, and it holds optimality and feasibility (see the detailed algorithm and proofs in [1]). It achieves transmission energy reduction by lowering the transmission power and increasing the transmission time as much as possible under the strictly convex and monotonically decreasing energyper-bit function. Now, since the energy-per-bit function is changed in our model, we develop a modified Lazy scheduling algorithm, which is an extended version of the Lazy scheduling. In order to devise modified Lazy scheduling, we consider the two lemmas induced from the trade-off relationship described in Section 2.

Lemma 1. There exists a packet transmission time $\tau_{\max }$, which minimizes the transmission energy consumption.

Proof. From Theorem 2, there exists a transmission time-per-bit $t^{*}$, which yields the minimum energy-per-bit value. Accordingly, we can obtain the packet 
transmission time $\tau_{\max }$, which minimizes the energy consumption needed to transmit a packet, by multiplying the packet size and $t^{*}$ together.

Lemma 1 tells us that we do not need to consider the range of $\tau$ longer than $\tau_{\max }$ in the optimization problem. The reason is that using $\tau\left(>\tau_{\max }\right)$ does not help minimizing the energy consumption nor satisfying the delay constraint.

Lemma 2. When the range of $\tau$, which we are interested in, is upper-bounded by $\tau_{\max }, w(\tau)$ can be regarded as a convex function of $\tau$.

Proof. According to Theorem 2] in Sect. 2, $E_{r}(t)$ is convex when the transmission time-per-bit $t$ is less than $t^{*}$ since $t^{*}$ is less than the inflection point $t^{o}$. Therefore, $w(\tau)$ is also convex in $\tau$ when $\tau<\tau_{\max }$.

Our modified Lazy scheduling algorithm consists of two parts. The first part is called minimum energy transmission part, where the scheduler finds the packets which can be transmitted with the minimum energy packet transmission time $\tau_{\max }$. The second part is called legacy Lazy scheduling part, where the Lazy scheduling is conducted for the set of the remaining packets. The modified algorithm is summarized in Algorithm 1 .

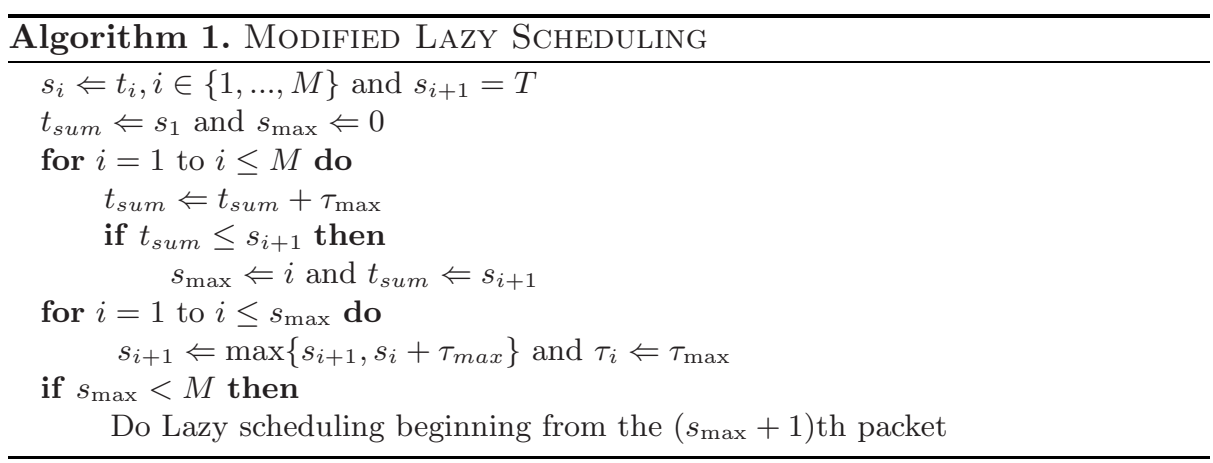

Modified Move Right Algorithm. In order to solve ESP-II, we modify the Move Right scheduling algorithm [10. Originally, Move Right algorithm was developed to solve ESP-I when the non-identical energy-per-bit transmission function is used for each packet. However, we modify this algorithm to deal with the constraints of per-packet delay bound. The main idea of the original Move Right algorithm is to find the optimal transmission start time with an iterative manner, and this notion of iteration is maintained in our proposed algorithm as well. The modified algorithm has two additional constraints: one is a delay bound for the feasibility and the other is a transmission duration bound for the minimum transmission energy. Initially, $s_{i}$ and $\tau_{i}$ are set to $t_{i}$ and $\min \left\{s_{i+1}-s_{i}, q_{i}-s_{i}, \tau_{\max }\right\}$, respectively. For a pair of packets, which arrive subsequently, we find $s_{2}{ }^{\prime}\left(s_{2} \leq s_{2}{ }^{\prime} \leq s_{2}+\tau_{2}\right), \tau_{1}{ }^{\prime}$, and $\tau_{2}{ }^{\prime}$ such that $w\left(\tau_{1}{ }^{\prime}\right)+w\left(\tau_{2}{ }^{\prime}\right)$ 
is minimized. This operation proceeds until the last two packets. In order to get the optimum value, the algorithm repeats this process until the scheduling results converge.

The detailed algorithm is described in Algorithm 2, where the superscript $k$ in $\tau_{i}^{k}$ and $s_{i}^{k}$ indicates the $k$ th iteration. $f\left(\tau_{i}^{k-1}, \tau_{i+1}^{k-1}, s_{i}^{k-1} s_{i+1}^{k-1}\right)$ returns the updated set of values $\left(\tau_{i}^{k}, \tau_{i+1}^{k}, s_{i+1}^{k}\right)$, which minimize $w\left(\tau_{i}^{k}\right)+w\left(\tau_{i+1}^{k}\right)$ when $\tau_{i}^{k-1}+\tau_{i+1}^{k-1}$ is fixed, where $\tau_{i}^{k}, \tau_{i+1}^{k}$, and $s_{i+1}^{k}$ satisfy $\tau_{i}^{k} \leq \tau_{\max }, \tau_{i+1}^{k} \leq \tau_{\max }$, and $s_{i+1}^{k} \leq s_{i+1}^{k-1}+\tau_{i+1}^{k-1} \leq \min \left\{s_{i+1}^{k}+\tau_{\max }, q_{i+1}\right\}$, respectively. If $s_{i+1}^{k-1}>s_{i}^{k-1}+\tau_{i}^{k-1}$, $\left(\tau_{i}^{k}, \tau_{i+1}^{k}, s_{i+1}^{k}\right)$ are not changed from the values at the $(k-1)$ th iteration. The optimality is also shown in [10] and this is maintained as well in our algorithm since we only consider the convex region of $w(\tau)$ given when $\tau$ is less than or equal to $\tau_{\max }$.

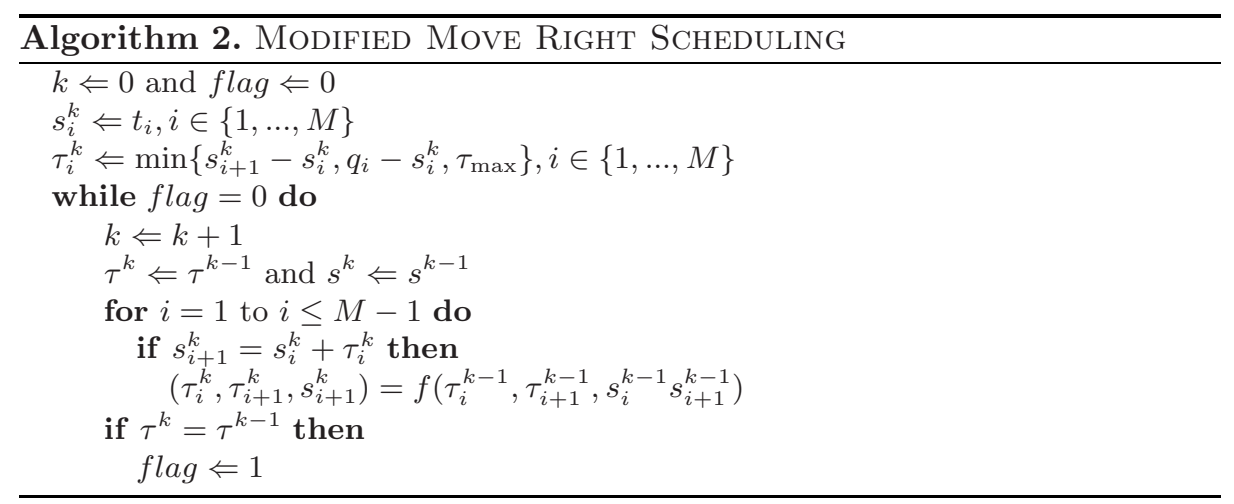

\subsection{Online Algorithm}

Online Extension of Modified Lazy Scheduling Algorithm. For the extension to online algorithm, we assume that packets arrive according to the Poisson distribution with mean rate $\lambda$. Our goal is to achieve the optimal performance in an average sense. To do so, we need a more tractable form of the scheduling result achieved by the offline algorithm and this was derived in 1 as $\tau_{j}{ }^{*}=\max _{k \in\left\{1, \ldots, M-\left(j+b_{j}\right)\right\}}\left\{\frac{1}{k+b_{j}} \sum_{i=1}^{k} C_{i}\right\}$, where $M$ is the total number of packets arriving during $[0, T), j$ is the current packet to be sent, and $C_{i}, i \in\left\{1, \ldots, M-j-b_{j}\right\}$ is the inter-arrival time between the $(j+i-1)$ th packet and the $(j+i)$ th packet. $b_{j}$ is the number of packets backlogged at that time when the $j$ th packet is transmitted. From this expression, one can derive a random variable $\tau^{*}(b, t)=\max _{k \in\{1, \ldots, M\}}\left\{\frac{1}{k+b} \sum_{i=1}^{k} D_{i}\right\}$, where $b$ is the current backlog, $M$ is a random variable representing the number of packet arrivals during $[t, T)$, and $D_{i}$ is the average inter-arrival time when $M=i$. Finally, the transmission duration of the current packet is determined by $E\left[\tau^{*}(b, t)\right]$. However, we simply modify it to $\tau^{*}=\min \left\{\tau_{\max }, E\left[\tau^{*}(b, t)\right]\right\}$ for our algorithm by considering $\tau_{\max }$. 
Online Extension of Modified Move Right Algorithm. Online extension of modified Move Right scheduling can also be achieved by the similar method used for the online modified Lazy scheduling case. In [10, the authors derived the online algorithm using a look-ahead buffer. In other words, the scheduler waits for gathering some packets during a determined time. However, for ESPI, this look-ahead buffer adds a constant value to the total transmission delay, and hence it can cause the actual duration of transmission to be shorter, which means that more energy consumption is required.

Accordingly, instead of using the notion of the look-ahead buffer, we make use of the assumption that for a given time interval, the packets arrive with the same inter-arrival time, which is determined by the ratio of the given time interval to the average number of packet arrivals during that interval. Then, we run Move Right scheduling to decide the transmit duration of the current packet using the information of $b$ backlogged packets and the expected packet arrivals, where each packet is assumed to have the same delay margin from its own arrival time.

\section{Simulation Results}

In this section, we evaluate the proposed algorithms using an MATLAB-based simulator. We assume that the size of a packet is $10 \mathrm{kbits}$ and the system bandwidth is $10^{6}$ symbols/sec. A simulation run lasts for $10 \mathrm{sec}$, and during the last $0.5 \mathrm{sec}$, no packet is assumed to arrive in order to prevent the transmission energy from divergence. For other parameters, we use the same values mentioned in Sect. 2. Whenever a result needs to be averaged, we repeat the simulation runs as many as 10 times.

First of all, we compare Lazy and modified Lazy scheduling for the same packet arrival patterns under the proposed trade-off model. As shown in Fig. 3 . when the arrival rate is less than 150 packets / sec, Lazy scheduling tends to send each packet with a transmit duration longer than $\tau_{\max }$, and hence more energy is consumed. In the meantime, modified Lazy scheduling limits the transmit duration up to $\tau_{\max }$, which yields the minimum energy consumption. However, as the packet arrival rate increases, the difference of energy-per-bit between two algorithms becomes marginal since both algorithms will yield the same result if the optimal transmit duration is less than $\tau_{\max }$.

Second, Fig. 4 compares the online modified Lazy scheduling algorithm with corresponding offline version. The discrepancy between two algorithms becomes larger as the packet arrival rate increases. This is due to the fact that the online algorithm only minimizes the average energy consumption.

Finally, Fig. 5 shows the energy per bit achieved by offline and online modified Move Right scheduling algorithm. In this simulation, we assume that the perpacket delay bound, $q_{i}$, is given by $200 \mathrm{msec}$ for every packet. When the packet arrival rate is less than 90 packets/sec, the delay bound does not affect the energy-per-bit much because the energy optimal transmit duration is determined as a value less than the delay bound. However, as the packet arrival rate increases, 


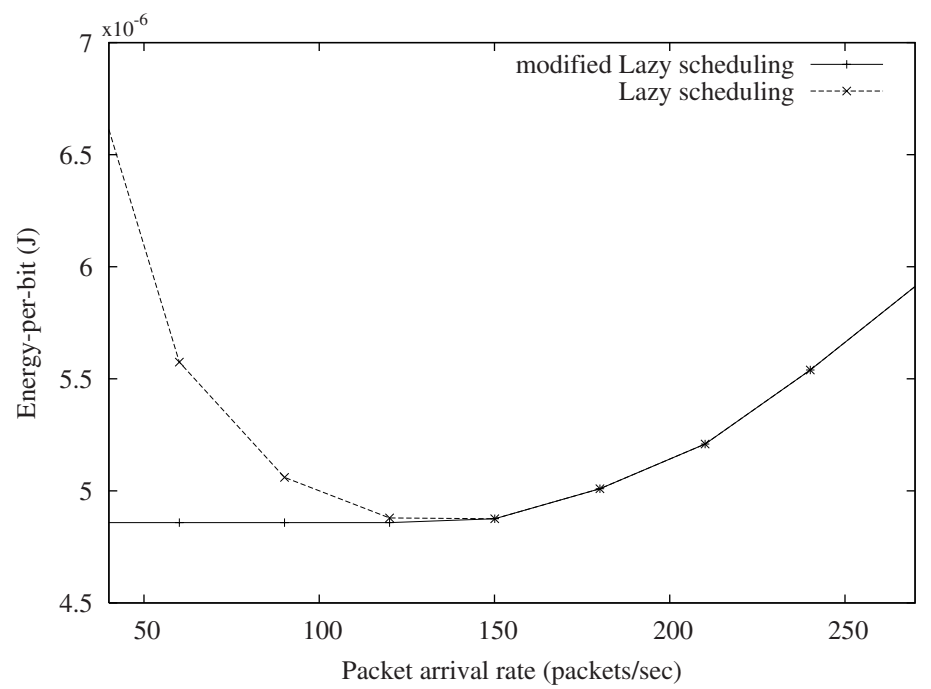

Fig. 3. Comparison of Lazy scheduling with modified Lazy scheduling

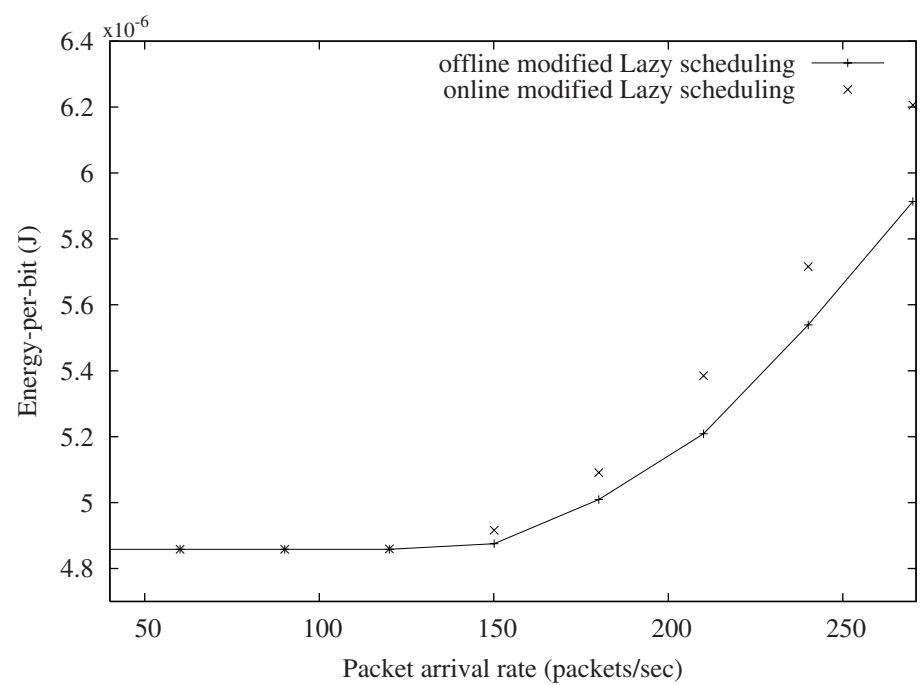

Fig. 4. Offline modified Lazy scheduling versus online modified Lazy scheduling

the delay bound tends to limit the transmit duration, and hence the energy-perbit also increases. This remark can be confirmed by comparing them with the modified Lazy scheduling result, which does not impose per-packet delay bound constraint. 


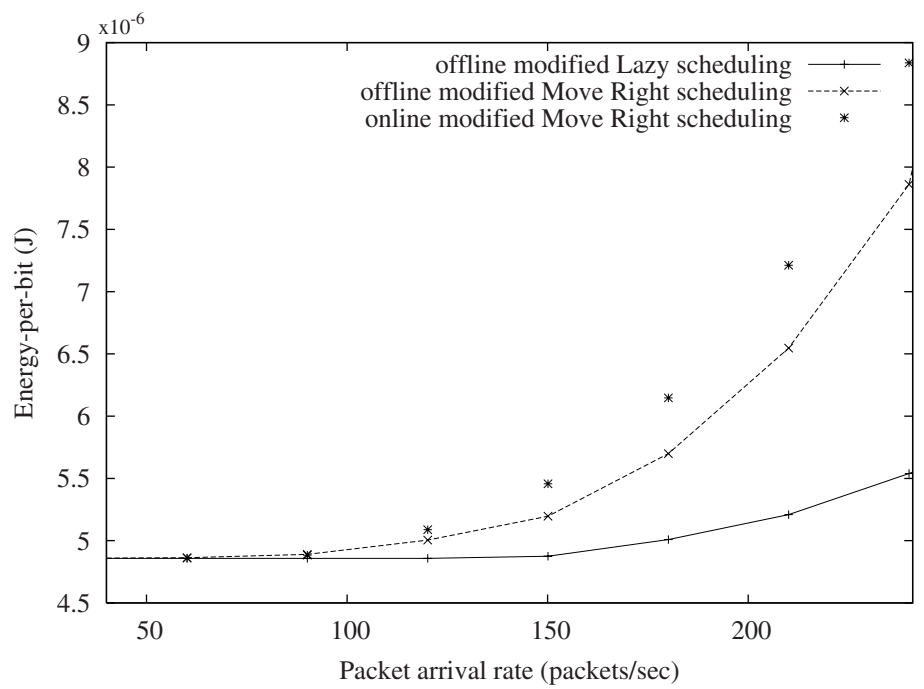

Fig. 5. Offline modified Move Right scheduling versus online modified Move Right scheduling

\section{Conclusion}

In this work, we show that the characteristic of power amplifier leads to a non-convex energy-per-bit curve. Based on this trade-off model, we propose modified Lazy scheduling and modified Move Right scheduling algorithms, which were originally proposed in 1, 10, to solve the energy minimization problem, where the delay constraint is imposed on either a group of packets or each packet. Since the transmit power in the uplink of a cellular network should be controlled in order to deal with the near-far problem and the inter-cell interference, it is our on-going work to solve both the energy-efficient scheduling and power control problem jointly in the multi-cell environment.

\section{References}

1. Prabhakar, B., et al.: Energy-Efficient Transmission over a Wireless Link via Lazy Packet Scheduling. In: Proc. IEEE INFOCOM. (April 2001)

2. Stemm, M., et al.: Reducing Power Consumption of Network Interfaces for Handheld Devices. In: Proc. MoMuC. (September 1996)

3. Flinn, J., Satyanarayanan, M.: Energy-aware adaptation for mobile applications. In: Proc. ACM SOSP. (December 1999)

4. Krashinsky, R., Balakrishnan, H.: Minimizing Energy for Wireless Web Access with Bounded Slowdown. In: Proc. ACM MobiCom. (September 2002)

5. Anand, M., et al.: Self-tuning wireless network power management. In: Proc. ACM MobiCom. (September 2003)

6. Qiao, D., Shin, K.G.: Smart Power-Saving Mode for IEEE 802.11 Wireless LANs. In: Proc. IEEE INFOCOM. (March 2005) 
7. Schurgers, C., et al.: Power Management for Energy-Aware Communication Systems. ACM Trans. Embedded Computing Sys. 2(3) (2003) 431-447

8. Yu, Y., et al.: Energy-Latency Tradeoffs for Data Gathering in Wireless Sensor Networks. In: Proc. IEEE INFOCOM. (March 2004)

9. Cui, S., et al.: Energy-constrained Modulation Optimization. IEEE Trans. Wireless Commun. 4(5) (2005)

10. Gamal, A.E., et al.: Energy-Efficient Scheduling of Packet Transmissions over a Wireless Networks. In: Proc. IEEE INFOCOM. (2002)

11. Jäger, H., et al.: Broadband High-Efficiency Monolithic InGap/GaAs HBT Power Amplifiers For 3G Handset Applications. IEEE MTT-S Inter. Microwave Sympo. Digest 2 (2002) 1035-1038

12. Pedro, J.C., et al.: Linearity versus Efficiency in Mobile Handset Power Amplifiers: A Battle without A Loser. Microwave Engineering Europe (August 2004)

13. Corte, F.D.: Power Management for Energy-Aware Communication Systems. RFDesign Magazine (May 2000) 